\title{
Diversity of iron oxidizers in wetland soils revealed by novel 16S rRNA primers targeting Gallionella-related bacteria
}

\author{
Juanjuan Wang ${ }^{1}$, Gerard Muyzer ${ }^{1,2}$, Paul LE Bodelier ${ }^{1}$ and Hendrikus J Laanbroek ${ }^{1}$ \\ ${ }^{1}$ Department of Microbial Wetland Ecology, Center for Limnology, Netherlands Institute of Ecology \\ (NIOO - KNAW), Maarssen, The Netherlands and ${ }^{2}$ Department of Biotechnology, Environmental \\ Biotechnology Group, Delft University of Technology, Delft, The Netherlands
}

\begin{abstract}
Neutrophilic iron-oxidizing bacteria (FeOB) are important catalysts of iron cycling in wetland environments. However, little is known about their diversity and distribution in various environments. The aim of this study was to develop a PCR-DGGE assay enabling the detection of neutrophilic iron oxidizers in wetland habitats. Gradient tubes were used to enrich FeOB. From these enrichments, a clone library was established on the basis of the almost complete 16S rRNA gene using the universal bacterial primers $27 \mathrm{f}$ and $1492 \mathrm{r}$. This clone library consisted of mainly $\alpha$ - and $\beta$-Proteobacteria, among which two major clusters were closely related to Gallionella spp. Specific probes and primers were developed on the basis of this 16S rRNA gene clone library. The newly designed Gallionella-specific 16S rRNA gene primer set 122f/998r was applied to community DNA obtained from three contrasting wetland environments, followed by Denaturing Gradient Gel Electrophoresis (DGGE) analysis. A second 16S rRNA gene clone library was constructed using the PCR products from one of our sampling sites amplified with the newly developed primer set 122 f/998r. The cloned 16S rRNA gene sequences all represented novel culturable iron oxidizers most closely related to Gallionella sp. On the basis of their nucleotide sequences, four groups could be identified that were comparable to the DGGE banding pattern obtained before with the same PCR products as used for the second clone library. Using these Gallionella-specific 16S rRNA genebased primers, in combination with DGGE, first insights into the diversity and distribution of these bacteria in wetland soils were obtained.
\end{abstract}

The ISME Journal (2009) 3, 715-725; doi:10.1038/ismej.2009.7; published online 19 February 2009

Subject Category: microbial ecology and functional diversity of natural habitats

Keywords: iron oxidation; Gallionella; wetlands; rhizosphere

\section{Introduction}

Iron is the fourth most abundant element in the Earth's crust and the most prevalent redox active metal (Hauck et al., 2001). It participates in many key biological processes, such as photosynthesis, $\mathrm{N}_{2}$ fixation, methanogenesis, $\mathrm{H}_{2}$ production and consumption, respiration, the trichloroacetic acid cycle, oxygen transport, gene regulation and DNA biosynthesis (Andrews et al., 2003). Iron also acts as an electron donor and acceptor for microbial energy generation. It has been the focus of many studies because of its importance in biogeochemical redox

Correspondence: J Wang, Department of Microbial Wetland Ecology, Center for Limnology, Netherlands Institute of Ecology (NIOO - KNAW), PO Box 1299, Maarssen 3600 BG, The Netherlands.

E-mail: j.wang@nioo.knaw.nl

Received 2 November 2008; revised 5 January 2009; accepted 5 January 2009; published online 19 February 2009 reactions in the environments (Straub et al., 2001; Emerson and Weiss, 2004; Roden, 2004).

Microorganisms can accelerate iron oxidation through a variety of mechanisms, such as changing local pH (Fortin et al., 1998), passive physicochemical sorption (Hallbeck and Pedersen, 1990; McLean et al., 1996; Ferris et al., 1999; Hanert, 2002), and enzymatic conversion of iron to oxidized forms (Ghiorse, 1984; Widdel et al., 1993). Iron-oxidizing bacteria $(\mathrm{FeOB})$ have been detected in a wide variety of environments and have been shown to increase the rate of iron oxidation by up to four orders of magnitude compared with the rate of strictly abiotic oxidation (Søgaard et al., 2001).

Compared with acidophilic iron oxidizers that have been studied extensively (Leduc and Ferroni, 1994; Auernik et al., 2008) much less is known about the eco-physiology of FeOB inhabiting environments of neutral $\mathrm{pH}$. This is largely due to the fact that their competitive ability in relation to abiotic oxidation still remains controversial (Sobolev and 
Roden, 2002), and to the difficulties associated with obtaining and maintaining them in pure culture (Edwards et al., 2003a). In the last decade, a number of studies (Hallbeck and Pedersen, 1995; Emerson and Moyer, 2002; Neubauer et al., 2002; Sobolev and Roden, 2002) showed that neutrophilic FeOB could successfully compete with abiotic oxidation for $\mathrm{Fe}$ (II); in some batch culture experiments, they accounted for $50-60 \%$ of the total Fe (II) oxidation.

The typical characteristic of these neutrophilic FeOB is their lithoautotrophic metabolism using iron as the only energy source. They are found growing at oxic-anoxic interfaces (Lutters-Czekalla, 1990) in neutral $\mathrm{pH}$ environments and are classified as micro-aerophilic bacteria. Among them, Gallionella ferruginea is known as the characteristic ironoxidizing bacterium (Engel and Hanert, 1967; Hanert, 1968; Hallbeck and Pedersen, 1991). The rhizosphere of wetland plants has been shown to be one of the niches for these organisms because of the fact that plant roots release excessive oxygen into the soil (Armstrong, 1964; Neubauer et al., 2007) creating suboxic conditions required by the iron oxidizers. Research on neutrophilic FeOB has recently moved forward with the isolation of a number of morphologically distinct species from different environments (Emerson and Moyer, 1997; Hanert, 2002; Edwards et al., 2003b, 2004; Sobolev and Roden, 2004; Weiss et al., 2007). Most of them were classified as $\beta$ - or $\gamma$-proteobacteria.

Despite the progress in isolation and the biogeochemical evidence supporting their potential role in Fe-cycling, knowledge concerning their diversity and environmental distribution is still lacking. In this study, FeOB were enriched and identified. A 16S rRNA gene clone library of the enrichments was constructed and specific probes and primers were designed subsequently. The distribution of FeOB in wetland environments was further investigated by the application of specific primers targeting the $16 \mathrm{~S}$ rRNA gene of Gallionella-related bacteria, in combination with PCR-DGGE, cloning and phylogenetic analysis.

\section{Materials and methods}

\section{Soil sampling}

Three sampling sites were chosen for this study. The first location, Appels (A; Belgium, $51{ }^{\circ} 2^{\prime} 0^{\prime \prime} \mathrm{N}, 4^{\circ} 4^{\prime}$ $0^{\prime \prime} \mathrm{E}$ ), is situated along the Scheldt estuary and has been characterized extensively (Lin et al., 2007). It is an intertidal freshwater marsh area typically colonized by Typha latifolia, Bolboschoenus maritimus and Phragmites australis. Waarde (W; The Netherlands, $51^{\circ} 25^{\prime} 2^{\prime \prime} \mathrm{N} 4^{\circ} 4^{\prime} 8^{\prime \prime} \mathrm{E}$ ) is a salt marsh where iron oxidation is very common and Spartina anglica is the dominating plant species. The sediments at Appels and Waarde contain significant amounts of ascorbate extractable Fe at near neutral $\mathrm{pH}$. The pore water Fe (II) concentration reached values as high as
$400 \mu \mathrm{M}$ (Hyacinthe and Van Cappellen, 2004; Lin et al., 2007). The third location, Huis Den Doorn (H; The Netherlands, $52^{\circ} 32^{\prime} 60^{\prime \prime} \mathrm{N}, 6^{\circ} 7^{\prime} 60^{\prime \prime} \mathrm{E}$ ), is an irregularly flooded inland wetland site in the center of the Netherlands, which has a remarkably high concentration of Fe (total Fe $702 \mu \mathrm{molg}^{-1}$ dry soil and oxalate-extracted Fe $459 \mu \mathrm{mol} \mathrm{g}^{-1}$ dry soil) (Loeb et al., 2008). Glyceria maxima is the dominating plant species.

Approximately $300 \mathrm{~g}$ of sieved soil (mesh size $1 \mathrm{~mm}$ ) from each sampling site was put in a glass beaker $(350 \mathrm{ml})$ and flooded with distilled water. One seedling (15 days old, two-leaf stage) of Glyceria maxima (for Appels and Huis den Doorn soil) or Spartina anglica (for Waarde soil) was transplanted into the glass beakers when the soil was completely saturated with water. Eight replicates for each soil type were prepared. The flood water level was kept constant at $2 \mathrm{~cm}$ above the soil surface by replenishing with sterile distilled water. After six weeks' growth at $20^{\circ} \mathrm{C}(16 \mathrm{~h} /$ light and 8 $\mathrm{h} /$ dark) and $70 \%$ humidity, the plants were harvested. Soil from $1 \mathrm{~cm}$ below the soil surface (T), rhizosphere (R) and bulk (B) sections were sampled separately. A portion of each sample was used for enrichment of FeOB. Other aliquots were freeze-dried and stored for further molecular analysis.

\section{Enrichment and isolation}

Fresh soil samples were mixed at a ratio of 1:1 with Milli-Q water and used as inocula for enrichment of bacteria. Plant roots containing visible iron plaques were rinsed in sterile deionized water to remove adhering soil particles, cut into sections and used directly for inoculation.

Gradient tubes with opposing gradients (Emerson et al., 1999) of oxygen and ferrous iron sulfide were used for the initial enrichment, isolation and subsequent maintenance of the culture. The system consisted of two layers of agarose in $16 \mathrm{~mm}$ screwcap glass tubes. A bottom layer contained $1.25 \mathrm{ml}$ FeS and Modified Wolfe Mineral Medium (Emerson and Floyd, 2005) at a ratio of 1:1, amended with $1 \%$ (wt/vol) agarose. The top layer consisted of $6.75 \mathrm{ml}$ mineral medium supplemented with vitamins and minerals (http://www.lgcstandards-atcc.org, MDVS, MD-TMS) and stabilized with $0.15 \%$ (wt/vol) agarose. Before autoclaving, the solutions for the upper layer were added together with $\mathrm{NaHCO}_{3}$ to a concentration of $0.5 \mathrm{mM} \mathrm{NaHCO}{ }_{3}$ and flushed with $\mathrm{CO}_{2}$ for $1 \mathrm{~min}$ to reach a final $\mathrm{pH}$ of 6.5 .

The gradient tubes were always inoculated $24 \mathrm{~h}$ after preparation to facilitate the diffusion of $\mathrm{Fe}^{2+}$ into the top layer where bacterial growth occurs. The tubes were inoculated by pulling a micropipette vertically through the medium while the inoculum was expelled. The inoculum was serially diluted in sterile Modified Wolfe Mineral Medium. Abiotic controls were prepared without bacteria. Positive cultures were identified by observation of a discrete 
band of $\mathrm{Fe}$ (III) oxide in contrast to the diffuse deposition of oxides in abiotic controls (Emerson and Moyer, 1997). The presence of bacteria was confirmed by both microscopy and molecular analysis.

\section{DNA extraction and PCR}

DNA was extracted using a modified DNA isolation procedure (Zhou et al., 1996). The purification of DNA was carried out with DNA Clean and concentrator TM kit (Zymo Research, Orange, CA, USA). The quantity and quality of the extracted DNA was analyzed by spectrophotometry using NanoDrop ND-1000 TM (Nano-Drop Technologies, Wilmington, DE, USA) and by agarose gel electrophoresis. The genomic DNA was stored at $-20^{\circ} \mathrm{C}$ for later use.

$16 S$ rRNA genes were amplified by PCR using different primer sets (see Table 1 for details).

\section{Denaturing Gradient Gel Electrophoresis (DGGE)}

Denaturing Gradient Gel Electrophoresis was performed with the Protean II system as described earlier (Muyzer et al., 1993). An 8\% polyacrylamide gel with a vertical gradient of $30-60 \%$ denaturants (100\% denaturants constitute a mixture of $7 \mathrm{M}$ urea and $40 \%$ (vol/vol) formamide) was used to analyze the $550 \mathrm{bp}$ PCR products. The running conditions were $100 \mathrm{~V}$ at a constant temperature of $60^{\circ} \mathrm{C}$ in $23 \mathrm{l}$ of $0.5 \times$ TAE buffer $(20 \mathrm{mM}$ Tris acetate, $0.5 \mathrm{mM}$ EDTA, pH 8.0) for $18 \mathrm{~h}$. The DGGE gels were visualized using a UV transilluminator after ethidium bromide staining. DNA fragments were excised from the gels, re-amplified, purified using the Gel DNA Recovery Kit (Zymoclean, Orange, CA, USA) and subjected to sequencing.

\section{Clone library of $16 S$ rRNA genes}

Two clone libraries were constructed in this experiment using the TA cloning kit (Invitrogen, Carlsbad, CA, USA, version V). For analyzing the gradient tube enrichments, cloning of nearly complete 16S rRNA gene fragments was performed. For the application of Gallionella-specific primers on environmental soil samples, another clone library was constructed with the PCR products obtained using the specific primers.

Ninety-six positive clones from each clone library were randomly picked for sequence analysis. The clones were inoculated into liquid LB medium amended with $20 \mathrm{mg} \mathrm{ml}^{-1}$ ampicillin and incubated on shakers at $37^{\circ} \mathrm{C}$ overnight. The cells were harvested and plasmid DNA was isolated using the Zyppy plasmid miniprep kit (Zymo Research). The plasmid DNA was sequenced in both directions using M13F/M13R primers (Table 1) by Macrogen (http://www.macrogen.co.kr).

\section{Comparative sequence analysis}

Sequences of both excised DGGE bands and clones were manually checked and modified with Sequencher software (Sequencher 4.1.4) and then compared with existing sequences using the online database of BLAST (http://www.ncbi.nlm.nih.gov/ BLAST/Blast). Sequences were aligned and phylogenetic trees were reconstructed in ARB (Ludwig et al., 2004; Pruesse et al., 2007) using the neighborjoining algorithm (http://www.arb-home.de/). Probes for sequences that are closely related phylogenetically to defined iron-oxidizing species were designed using the probe design tool from the ARB package. The newly designed probes were aligned against sequences from the online sequence database (http://rdp.cme.msu.edu/probematch/search.jsp), and seven with less than 2 mismatches were chosen as specific probes.

On the basis of these probes, three primer sets were designed, which were 122F/998R, 122F/997R and $645 \mathrm{~F} / 996 \mathrm{R}$. Primers $122 \mathrm{~F}$ and $998 \mathrm{R}$, specific to Gallionella-related FeOB, were selected for further analysis. The PCR conditions were optimized by running gradient PCRs at different annealing temperatures $\left(50.3,51.9,53,54.3,55\right.$ and $\left.58^{\circ} \mathrm{C}\right)$ and $55{ }^{\circ} \mathrm{C}$ was found to yield products with the best quality and was used for this newly designed primer set. To test the specificity of the primers, plasmid DNA of clones of almost complete 16S rRNA gene sequences from non-related species (that is, MWE_C17, MWE_C40, MWE_N10, MWE_N19, MWE_C10 in Figure 4) were used as templates.

Table 1 16S rRNA gene PCR primers used in this study

\begin{tabular}{llll}
\hline Primer & Sequence $\left(5^{\prime}-3^{\prime}\right)$ & Application & Reference \\
\hline 341F-GC & CCTACGGGAGGCAGCAG & DGGE analysis & Muyzer et al. (1993) \\
907R & CCGTCAATTCMTTTGAGTTT & & Muyzer et al. (1993) \\
27F & GTGCTGCAGAGAGTTTGTCCTGGCTCAG & Clone library of gradient tube enrichments & Lane (1991) \\
1492R & CACGGATCCTACGGGTACCTTGTTACGACTT & & Lane (1991) \\
M13F & GTAAAACGACGGCCAG & Sequencing DNA from clones & Huey and Hall (1989) \\
M13R & CAGGAAACAGCTATGAC & Detecting Gallionella-like organisms in soil samples & This study \\
122F & ATATCGGAACATGTCCGG & & This study \\
998R & CTCTGGAAACTTCCTGAC & &
\end{tabular}




\section{Results}

Enrichment of $\mathrm{FeOB}$

Enrichment experiments started in winter time when no plant growth occurs in the field. Hence, plants were grown in growth chambers to enable differentiation between surface layers, rhizosphere and bulk soils. After 6 weeks of growth under controlled conditions, the soils in the beakers were fully rooted. Deposits of oxidized iron could be seen both around the roots and close to the soil surface (Figure 1a). Three days after inoculation of the soil samples into the gradient tubes, all the inoculated tubes showed growth. In each tube, one brownish iron oxide band mixed with bacterial biomass was formed approximately $1 \mathrm{~cm}$ below the surface. It was defined as growth because the shape and position of iron oxides were distinct from those in non-inoculated controls (as shown in Figure 1b). In the tubes inoculated with bulk or rhizosphere soil, similar iron oxide band patterns had been formed, whereas in tubes inoculated with fresh roots, the oxide band developed close to the headspace. Microscopic test and DNA isolation further confirmed bacterial growth associated with the iron oxide bands.

It was expected that pure cultures would be obtained after a few transfers, as described by Emerson and Moyer, (1997). However, in our case, a mixture of several species remained together till the last stage of isolation. Among them, one was closely related to $G$. ferruginea on the basis of the $16 \mathrm{~S}$ rRNA gene information (96\% sequence identity). With the increase in transfer times, the browncolored iron bands developed much slower. It took approximately $24 \mathrm{~h}$ to see a bright orange iron band at the beginning of the enrichment, whereas after a few transfers, it took several days to form a thick white band. A brown color was observed only a week later. Typically, these bands were thinner and tended to move deeper toward the bottom of the tubes compared with the early gradient cultures.

\section{DGGE analysis of enrichment cultures}

The cultures obtained in the gradient tube during the first transfers were analyzed using PCR-DGGE on the basis of general bacterial primers. On average, 15 different DGGE bands were detected in the enrichments (Figure 2a), which were excised and sequenced. The sequences were quite diverse, including different species of $\alpha$ - and $\beta$-Proteobacteria, and Bacteroidetes as well as Firmicutes (Figure 3). After another four transfers, the total number of DGGE bands diminished in each of the samples (for example, Figure 2b).

\section{Clone library}

The enrichments from the gradient tubes (5-6 transfers of AT, HB and HT) were pooled and used
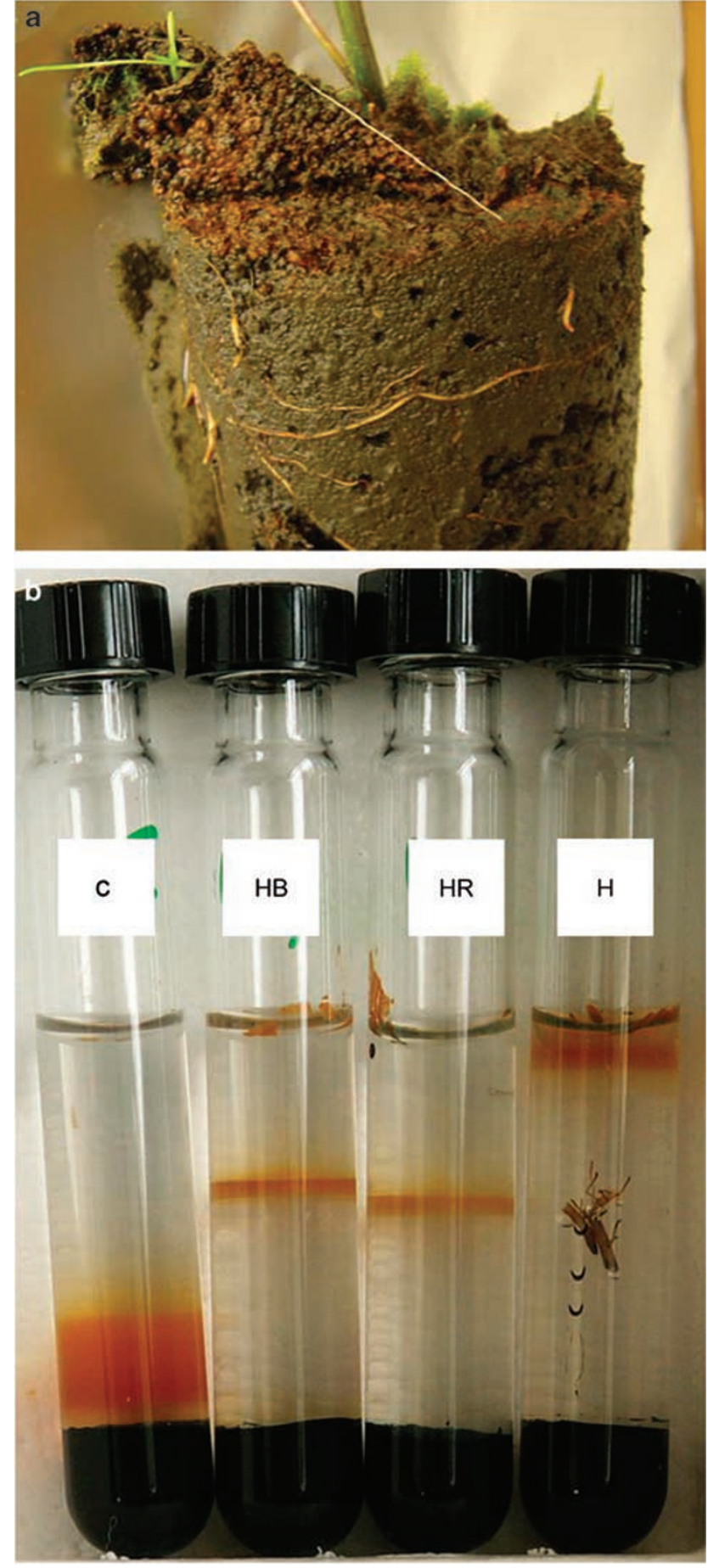

Figure 1 (a) Iron oxidation at the soil surface and around the roots of wetland plants. (b) Gradient tubes showing bacterial (HB, HR, H) and chemical iron oxidation (C). HB: Huis den Doorn bulk soil, HR: Huis den Doorn rhizosphere soil, H: Huis den Doorn root inoculum.

for DNA isolation and cloning. In total, 96 clones were screened. Figure 4 shows the phylogenetic affiliation of the different clone types (coded MWE) found in the enrichments. In the clone library, over $20 \%$ of the clones were closely related to G. ferruginea (Hallbeck et al., 1993), which is a 


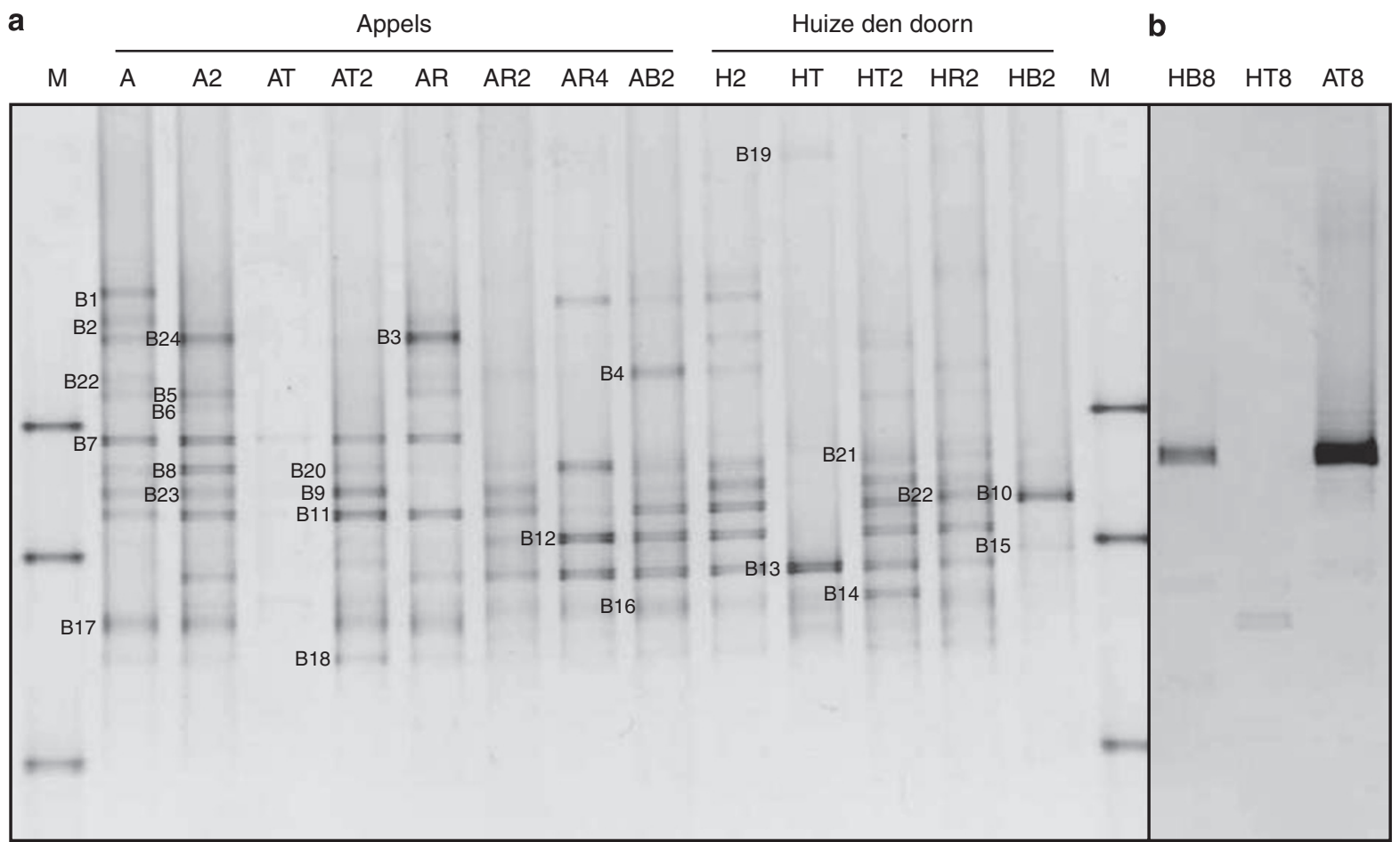

Figure 2 Denaturing gradient gel electrophoresis (DGGE) patterns showing diverse bacterial communities after the first (a) and later (b) transfers in gradient tube enrichments. A and $\mathrm{H}$ refer to the sampling sites Appels and Huis Den Doorn, respectively; B, T and M indicate bulk soil, surface soil and markers, respectively; numbers refer to numbers of transferring times. B1-B24 are cut and sequenced bands, and the sequence information can be found in Figure 3.

typical neutrophilic iron oxidizer (Hallberg and Ferris, 2004). They clustered either with Siderooxydans lithotrophicus LD-1 and Siderooxydans lithoautotrophicus ES-1, which are two recently identified iron oxidizers (Weiss et al., 2007), or with uncultured Gallionellaceae (Winderl et al., 2008).

Apart from the iron oxidizer group, in the clone library, we also found sequences that are related to other genera such as Azospira, Rhodocylclaceae, Janthinobacterium, Thiobacillus (Figure 4).

Design of specific primers and probes

Sequences from three clusters of interest (see arrows in Figure 4), including six representative clones on the bases of their assumed iron-oxidizing capacity, were chosen for specific probe or primer development. MWE_C19, MWE_N34, MWE_N10 and MWE_C13 fall into the Gallionella group. MWE_C19 and MWE_N34 share 95\% similarity to $G$. ferruginea and $98 \%$, similar to Gallionella capsiferriformans (Weiss et al., 2007), whereas MWE_N10 and MWE_C13 are closely related to Siderooxydans species. MWE_C10, MWE_C12 are related to some uncultured $\beta$-Proteobacterium found in iron-rich areas (Juretschko et al., 2002). In total, seven probes (that is, P1-P7) were developed and their specificity was checked using a probe-matching program (Table 2). Finally, three primer sets were designed on the basis of the probes, that is, $122 \mathrm{~F} / 998 \mathrm{R}, 122 \mathrm{~F} /$ 997R and 645F/997R. Primer set $122 \mathrm{~F} / 998 \mathrm{R}$ is specific to two almost identical sequences that share $98 \%$ similarity to a recently described iron-oxidizing bacterium Gallionella capsiferriformans on the basis of the 16S rRNA gene. Clones MWE_N10 and MWE_C13, clustering with Siderooxydans spp., share the same forward primer $122 \mathrm{~F}$ with clones MWE C19 and MWE N34, but have their own specific reverse primer 997R. The third primer set 645F/997R was designed for clones MWE_C10 and MWE_C12, which have uncultured Rhodocyclaceae spp. as their closest relative.

Detection of Gallionella-like bacteria in soil samples Confirmed to be specific for one novel cluster of culturable iron oxidizers, the primer set 122f/998r designed for Gallionella-like organisms was then used to detect these organisms in environmental samples. After a nested-PCR using the universal Eubacterial primer set 3G/907R, the PCR products were checked on DGGE gel. The result showed that these organisms were present in most of the soil samples tested (Figure 5). The bands migrated to or around the position of the marker band that belongs to the clone on which the primers were designed (Marker c in Figure 5). Differences in band patterns can be seen among the samples. As to the samples from Huis den Doorn, no bands were detected from the bulk soil (HB) and rhizosphere (HR), whereas the most intensive band was obtained in the surface soil (HT). Appels soil sample (AB, AR and AT) appeared 


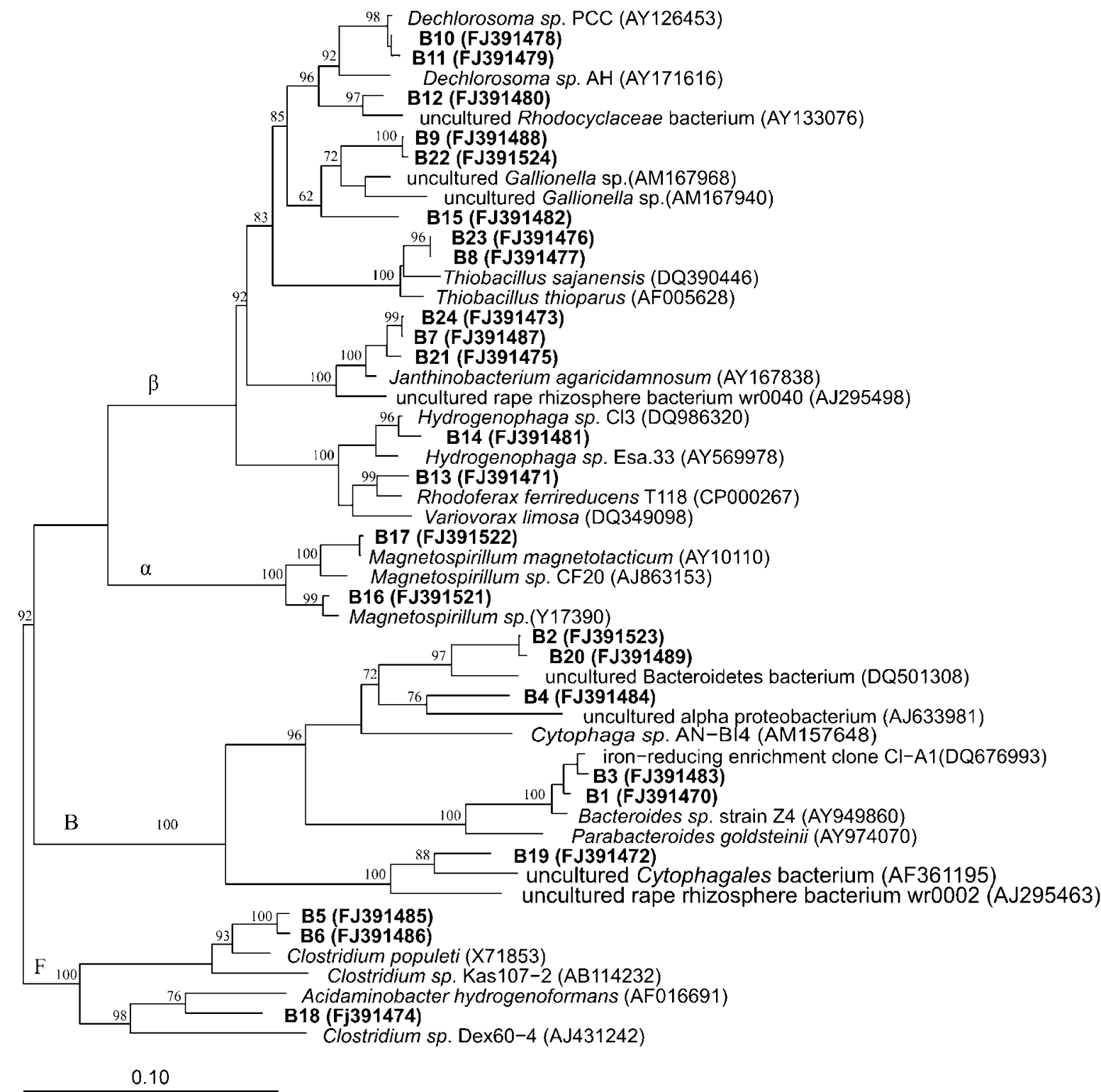

Figure 3 Neighbor-joining tree of sequences obtained from gradient tube enrichments shown in Figure 2a. Sequences determined in this study are printed in bold type. Bootstrap values are shown near the nodes (based on 1000 replicate trees). The bar indicates $10 \%$ sequence difference. Thermotoga maritima was used as an outgroup, but was pruned from the tree. $\alpha=$ Alphaproteobacteria, $\beta=$ Betaproteobacteria, $\mathrm{B}=$ Bacteroidetes, $\mathrm{F}=$ Firmicutes.

to be more diverse with respect to the presence of Gallionella-like bacteria than the other two soils.

To validate the PCR-DGGE method, a clone library was constructed using the PCR products of the Gallionella-specific primers (122F and 998R). Soil DNA was taken from one of the sampling sites, Huis den Doorn. Within 96 sequenced clones retrieved from these soil samples, four clusters could be identified for their differences in nucleotide sequences. Twelve sequences were chosen as representatives to be included in the phylogenetic tree on the basis of the clones from the gradient tube enrichments (Figure 4). Compared with the other clones, Hc8 is less closely related to G. ferruginea and other clones. Clones Hc37, Hc11 and Hc16 are identical to some uncultured Gallionella spp., whereas clones Hc9, Hc25, Hc30 and Hc1 share more similar sequences. Clones Hc18, Hc46 and Hc38 shared $97 \%$ similarity with $G$. ferruginea. By comparing the obtained sequences, the most discriminative variable region could be identified, which corresponds to Escherichia coli 16S rRNA range 455-479. These 12 representative sequences were checked again on DGGE (Figure 6). According to their migrating positions in the gradient gel, different groups could be distinguished. This was compared with the result obtained with the nested PCR-DGGE method (Lane HT in Figure 5). As expected, more 


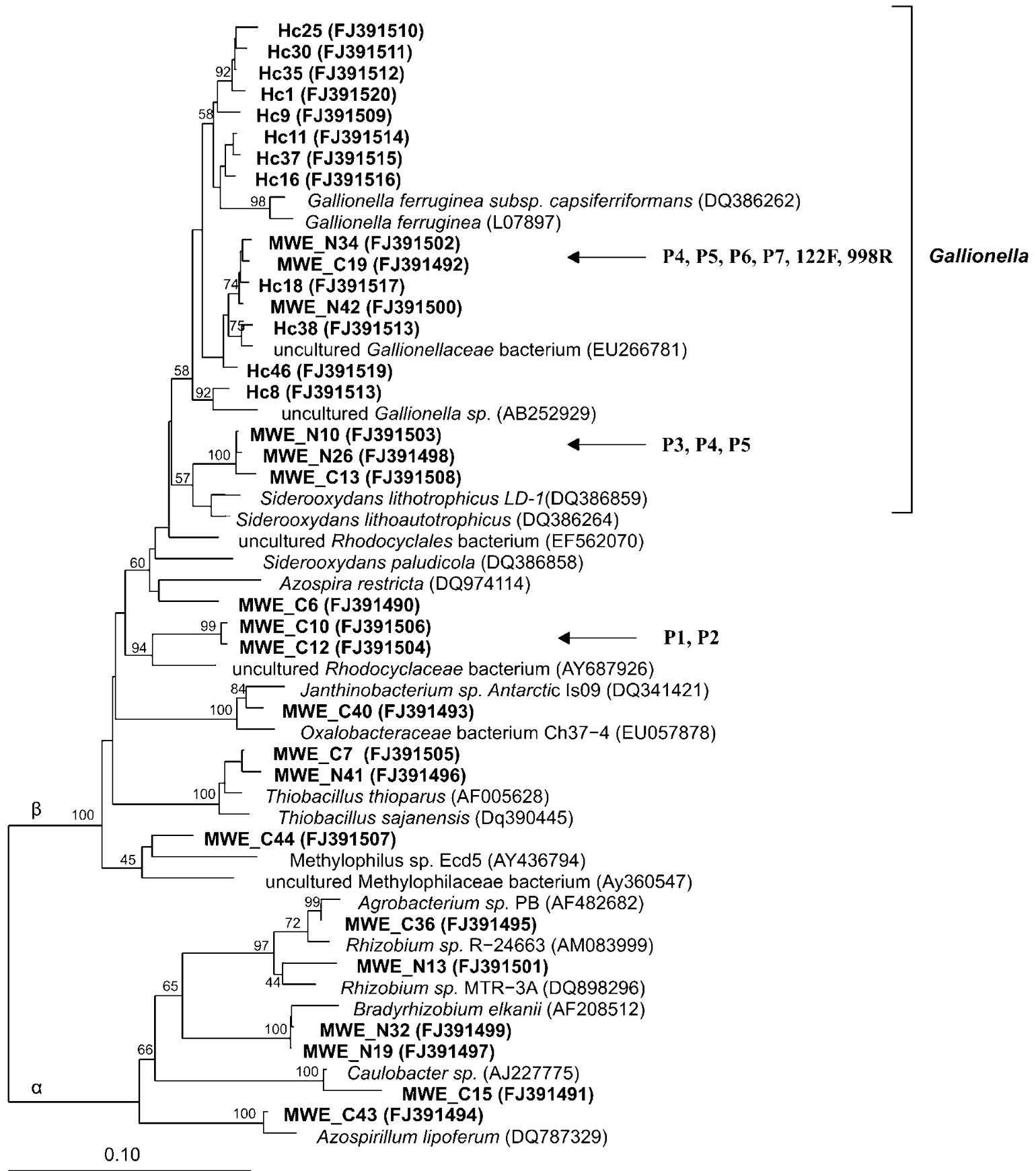

Figure 4 Phylogenetic tree of 16S rRNA-based clones derived from gradient tube enrichments using the 27f/1492r primer set (coded MWE), as well as from iron-rich Huis den Doorn soil samples using primer 122F/998R developed in this study (coded Hc). The sequences in bold were selected clones. Bootstrap values are shown near the knots (based on 1000 replicate trees). The bar indicates $10 \%$ sequence difference. Thermotoga maritima was used as an outgroup, but was pruned from the tree. $\alpha=$ Alphaproteobacteria, $\beta=$ Betaproteobacteria. Arrows indicate the clones based on which specific probes and primers developed.

bands were obtained after cloning, but most of them ended up at the same positions.

\section{Discussion}

Microbial iron oxidation under neutral conditions has been reported earlier (Hallbeck and Pedersen, 1990; Straub et al., 2001; Sobolev and Roden, 2002), but progress in this research area is hampered by the difficulty of obtaining the responsible organisms in pure culture. The development of the gradient tube technique (Emerson and Floyd, 2005) made it possible to obtain these bacteria in pure cultures. In this study, it was observed that when fresh roots were used as inoculation, they formed iron oxide bands close to the headspace where the oxygen concentration is relatively high. This is probably due to the depletion of 
Table 2 Specific 16S rRNA-targeted oligonucleotide probes designed according to the clone library from the mixed enrichment cultures of the gradient tubes

\begin{tabular}{|c|c|c|c|}
\hline Probe name & Probe Sequence $\left(5^{\prime}-3^{\prime}\right)$ & Position & Clone specificity \\
\hline P1_996 & CAGCAGGATTCCAGGCAT & 996-1013 & MWE_C10, MWE_C12 \\
\hline P2_645 & GCCACACTCGAGTCTTGC & $645-662$ & MWE_C10, MWE_C12 \\
\hline P3_997 & TCTTCTGGATTCTCGGCA & 997-1014 & MWE_N10, MWE_C13 \\
\hline P4_460 & CACTCCACGTATTAGGTG & $460-477$ & MWE_N10, MWE_C13 \\
\hline P5_122 & CCGGACATGTTCCGATAT & $122-140$ & $\begin{array}{l}\text { MWE_C19, MWE_N34 } \\
\text { MWE_N10, MWE_C13 }\end{array}$ \\
\hline P6_645 & GCTACACTCTAGCCTTCC & $645-662$ & MWE_C19, MWE_N34 \\
\hline P7_998 & CTCTGGAAACTTCCTGAC & 998-1015 & MWE_C19, MWE_N34 \\
\hline
\end{tabular}

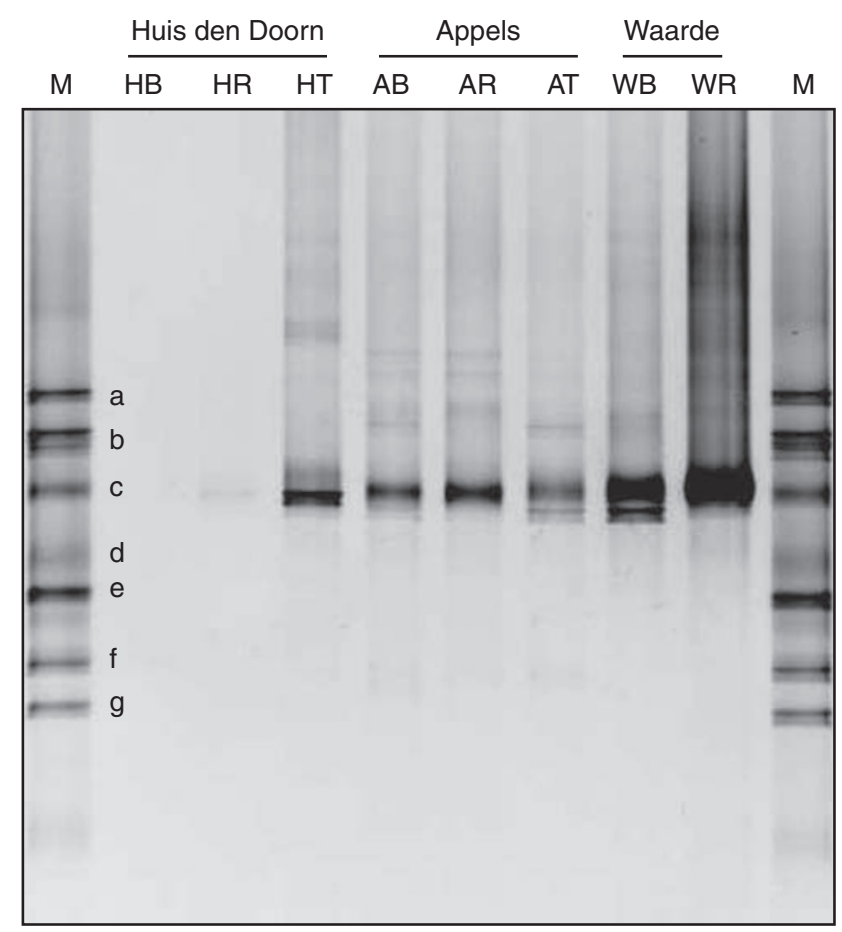

Figure 5 Denaturing gradient gel electrophoresis (DGGE) patterns showing DNA of Gallionella-like iron oxidizers from different wetland environments being amplified by using a nested approach (Gallionella-specific primers and universal DGGE primers 341GC/907R, respectively). A and $\mathrm{H}$ refer to the sampling sites Appels and Huis Den Doorn, respectively; B, T and M indicate bulk soil, surface soil and markers, respectively. Marker sequences are clones from the library of the gradient tube enrichments; from a to g: MWE_C40, MWE_N41, MWE_N34, MWE_N13, MWE_N19, MWE_C13 and MWE_N26 (see also Figure 4). Marker c indicates the clone from which the Gallionella-specific primers are developed.

oxygen by root respiration, which results in the upward movement of micro-aerophilic bacteria.

The success of isolation depends in large part on experience. In our study, the repeated dilution and inoculation of gradient tubes reduced the bacterial diversity significantly (as can be seen comparing Figures 2a and b). With the increase of the time between transfers, the iron oxide bands in the gradient tubes developed much slower and were thinner, which might be the consequence of a decrease in the number of many non-dominant
FeOB, as well as of those microorganisms that might benefit the growth of the remaining iron-oxidizing species. A pure iron-oxidizing isolate was obtained in this study, which shared the same 16S rRNA sequences as the clone used for designing the Gallionella-specific primers.

Despite its advantages of accuracy and efficiency, molecular tools are hardly applied in the study of FeOB in environments of approximately neutral $\mathrm{pH}$, mainly because of the lack of sequence information to design specific primers or probes for conservative regions of the 16S RNA genes (Konstantinidis and Tiedje, 2007) as well as for functional genes (Parro et al., 2007). We developed $16 \mathrm{~S}$ rRNA gene-based probes and primers, which can be used directly on environmental samples to study the occurrence of these microbes and their temporal and spatial distribution. In combination with laboratory cultivation, this opens up the ecological studies on this environmentally relevant group of bacteria.

By using a nested PCR-DGGE approach, we were able to detect Gallionella-like organisms in different compartments of freshwater as well as brackish wetland environments. As shown in Figure 5, the bands ended up at slightly different positions in DGGE gels, showing that the diversity of Gallionellarelated iron oxidizers is higher than recently known. The slightly higher diversity revealed by cloning compared with a nested PCR-DGGE approach showed that cloning has decreased the detection limit as compared with DGGE. In fact, in this study, the species detected by cloning, but missed by DGGE, that is, Hc8, Hc9 and Hc25, count for only 5\% of the total clones obtained. Hc8 is the only one out of the 96 clones that are distantly related to the other sequences. The low density of this species might increase the difficulty in detecting it by DGGE.

It was reported that in wetland environments iron oxidation mainly occurs close to the plant roots where sufficient oxygen is available owing to its release (Neubauer et al., 2007). We have indeed detected Gallionella-related organisms not only in the rhizosphere but also in the bulk soil. Detection based on the 16S rRNA gene indicates the presence but not the activity of microbial cells. Hence, cells detected in the rhizosphere may still be more active than those found in the bulk soil. 


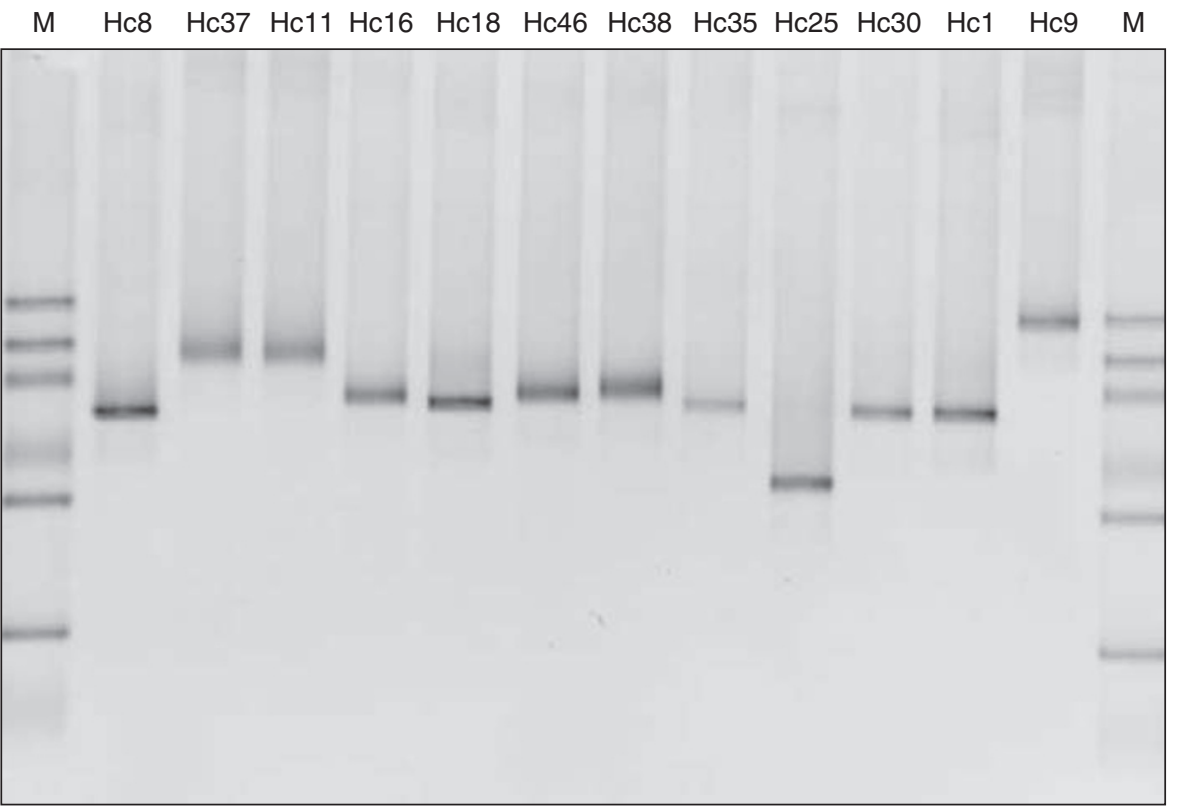

Figure 6 Denaturing gradient gel electrophoresis (DGGE) patterns showing cloned sequences amplified with the Gallionella-specific primers $122 \mathrm{~F}$ and 998R. Corresponding clones can be found in Figure 4. Hc refer to the codes of the clones, and $\mathrm{M}$ is the marker (same as Figure 5).

Iron oxidizers are phylogenetically so diverse that they fall in evolutionarily distinct groups (Roden et al., 2004). Beside the facts that not many culturable $\mathrm{FeOB}$ are available, the known sequences are so diverse that it is almost not possible to design group-specific probes or primers for them. With our specific primers, four different groups were selectively picked up from environmental wetland samples. The comparison of sequences showed that they vary in a specific region. Therefore, primers can be developed to detect Gallionellarelated sequences separately from each other using real time-PCR.

To our knowledge, this is the first study using specific primers for detecting neutrophilic $\mathrm{FeOB}$ in environmental samples. Although bacterial iron oxidation has been claimed to be important especially in wetland environments (Neubauer et al., 2007), studies on this subject are still limited. The lack of efficient molecular tools to study these microbes seriously hampers progress in this field. This fact will make the detection of their distributions in the environments a challenge. Isolation of pure cultures and sequencing of their genomes will help to develop and define primers on the basis of functional genes, which will also facilitate mRNA studies on these microbes.

\section{Acknowledgements}

We thank Dr Marzia Milletto for providing Huis Den Doorn soil samples. Dr Jeanine Geelhoed is acknowledged for sharing her experience with FeOB. This work was financially supported by the Netherlands Darwin Center for Biogeology (Grant number 142.16.1031).

\section{References}

Andrews SC, Robinson AK, Rodriguez-Quinones F. (2003). Bacterial iron homeostasis. FEMS Microbiol Rev 27: 215-237.

Armstrong W. (1964). Oxygen diffusion from roots of some British bog plants. Nature 204: 801-802.

Auernik KS, Maezato Y, Blum PH, Kelly RM. (2008). The genome sequence of the metal-mobilizing, extremely thermoacidophilic archaeon Metallosphaera sedula provides insights into bioleaching-associated metabolism. Appl Environ Microbiol 74: 682-692.

Edwards KJ, Bach W, McCollom TM, Rogers DR. (2004). Neutrophilic iron-oxidizing bacteria in the ocean: their habitats, diversity, and roles in mineral deposition, rock alteration, and biomass production in the deep-sea. Geomicrobiol J 21: 393-404.

Edwards KJ, Bach W, Rogers DR. (2003a). Geomicrobiology of the ocean crust: a role for chemoautotrophic Fe-bacteria. Biol Bull 204: 180-185.

Edwards KJ, Rogers DR, Wirsen CO, McCollom TM. (2003b). Isolation and characterization of novel psychrophilic, neutrophilic, Fe-oxidizing, chemolithoautotrophic $\alpha$ - and $\gamma$-Proteobacteria from the deep sea. Appl Environ Microbiol 69: 2906-2913.

Emerson D, Floyd MM. (2005). Enrichment and isolation of iron-oxidizing bacteria at neutral $\mathrm{pH}$. Methods Enzymol 397: 112-123.

Emerson D, Moyer C. (1997). Isolation and characterization of novel iron-oxidizing bacteria that grow at circumneutral pH. Appl Environ Microbiol 63: 4784-4792.

Emerson D, Moyer CL. (2002). Neutrophilic Fe-oxidizing bacteria are abundant at the Loihi seamount hydrothermal vents and play a major role in $\mathrm{Fe}$ oxide deposition. Appl Environ Microbiol 68: 3085-3093.

Emerson D, Weiss JV. (2004). Bacterial iron oxidation in circumneutral freshwater habitats: findings from the field and the laboratory. Geomicrobiol J 21: 405-414. 
Emerson D, Weiss JV, Megonigal JP. (1999). Iron-oxidizing bacteria are associated with ferric hydroxide precipitates (Fe-plaque) on the roots of wetland plants. Appl Environ Microbiol 65: 2758-2761.

Engel H, Hanert H. (1967). Isolierung von Gallionella ferruginea ehrenberg. Naturwissenschaften 54: 147.

Ferris FG, Konhauser KO, Lyven B, Pedersen K. (1999). Accumulation of metals by bacteriogenic iron oxides in a subterranean environment. Geomicrobiol J 16: 181-192.

Fortin D, Ferris FG, Scott SD. (1998). Formation of Fesilicates and Fe-oxides on bacterial surfaces in samples collected near hydrothermal vents on the Southern Explorer Ridge in the northeast Pacific ocean. American Mineralogist 83: 1399-1408.

Ghiorse WC. (1984). Biology of iron- and manganesedepositing bacteria. Annul rev microbiol 38: 515.

Hallbeck L, Pedersen K. (1990). Culture parameters regulating stalk formation and growth-rate of Gallionella-ferruginea. J Gen Microbiol 136: 1675-1680.

Hallbeck L, Pedersen K. (1991). Autotrophic and mixotrophic growth of Gallionella ferruginea. J Gen Microbiol 137: 2657-2661.

Hallbeck L, Pedersen K. (1995). Benefits associated with the stalk of Gallionella ferruginea, evaluated by comparison of stalk-forming and a non-stalk forming strain and biofilm studies in-situ. Micro Ecol 30: 257-268.

Hallbeck L, Stahl F, Pedersen K. (1993). Phylogeny and phenotypic characterization of the stalk-forming and iron-oxidizing bacterium Gallionella ferruginea. J Gen Microbiol 139: 1531-1535.

Hallberg R, Ferris FG. (2004). Biomineralization by Gallionella. Geomicrobiol J 21: 325-330.

Hanert H. (1968). Investigations on isolation physiology and morphology of Gallionella ferruginea ehernberg. Archiv Fur Mikrobiologie 60: 348-376.

Hanert HH. (2002). Bacterial and chemical iron oxide deposition in a shallow bay on Palaea Kameni, Santorini, Greece: microscopy, electron probe microanalysis, and photometry of in situ experiments. Geomicrobiol J 19: 317-342.

Hauck S, Benz M, Brune A, Schink B. (2001). Ferrous iron oxidation by denitrifying bacteria in profundal sediments of a deep lake (Lake Constance). FEMS Microbiol Ecol 37: 127-134.

Huey B, Hall J. (1989). Hypervariable DNA fingerprinting in Escherichia Coli: minisatellite probe from bacteriophage-M13. J Bacteriol 171: 2528-2532.

Hyacinthe C, Van Cappellen P. (2004). An authigenic iron phosphate phase in estuarine sediments: composition, formation and chemical reactivity. Mar Chem 91: 227-251.

Juretschko S, Loy A, Lehner A, Wagner M. (2002). The microbial community composition of a nitrifyingdenitrifying activated sludge from an industrial sewage treatment plant analyzed by the full-cycle rRNA approach. Syst Appl Microbiol 25: 84-99.

Konstantinidis KT, Tiedje JM. (2007). Prokaryotic taxonomy and phylogeny in the genomic era: advancements and challenges ahead. Curr Opin in Microbiol 10: 504-509.

Lane DJ. (1991). 16S/23S rRNA sequencing. In: E Stackebrandt, M Goodfellow (eds). Nucleic Acid Techniques in Bacterial Systematics. Wiley: New York, pp 115-175.

Leduc LG, Ferroni GD. (1994). The chemolithotrophic bacterium Thiobacillus ferrooxidans. FEMS Microbiol 14: 103-119.
Lin B, Hyacinthe C, Bonneville S, Braster M, Van Cappellen P, Roling WFM. (2007). Phylogenetic and physiological diversity of dissimilatory ferric iron reducers in sediments of the polluted Scheldt estuary, Northwest Europe. Environ Microbiol 9: 1956-1968.

Loeb R, Lamers LP, Roelofs JG. (2008). Prediction of phosphorus mobilisation in inundated floodplain soils. Environ Pollut 156: 325-331.

Ludwig W, Strunk O, Westram R, Richter L, Meier H, Yadhukumar A et al. (2004). ARB: a software environment for sequence data. Nucleic Acids Res 32: 1363-1371.

Lutters-Czekalla S. (1990). Lithoautotrophic growth of the iron bacterium Gallionella ferruginea with thiosulfate or sulfide as energy source. Arch Microbiol 154: 417-421.

McLean RJC, Fortin D, Brown DA. (1996). Microbial metalbinding mechanisms and their relation to nuclear waste disposal. Can J Microbiol 42: 392-400.

Muyzer G, Dewaal EC, Uitterlinden AG. (1993). Profiling of complex microbial-populations by Denaturing Gradient Gel-Electrophoresis analysis of polymerase chain reaction-amplified genes-coding for $16 \mathrm{~S}$ ribosomal-RNA. Appl Environ Microbiol 59: 695-700.

Neubauer SC, Emerson D, Megonigal JP. (2002). Life at the energetic edge: kinetics of circumneutral iron oxidation by lithotrophic iron-oxidizing bacteria isolated from the wetland-plant rhizosphere. Appl Environ Microbiol 68: 3988-3995.

Neubauer SC, Toledo-Duran GE, Emerson D, Megonigal JP. (2007). Returning to their roots: iron-oxidizing bacteria enhance short-term plaque formation in the wetlandplant rhizosphere. Geomicrobiol J 24: 65-73.

Parro V, Moreno-Paz M, Gonzalez-Toril E. (2007). Analysis of environmental transcriptomes by DNA microarrays. Environ Microbiol 9: 453-464.

Pruesse E, Quast C, Knittel K, Fuchs BM, Ludwig WG, Peplies J et al. (2007). SILVA: a comprehensive online resource for quality checked and aligned ribosomal RNA sequence data compatible with ARB. Nucleic Acids Res 35: 7188-7196.

Roden EE. (2004). Analysis of long-term bacterial vs chemical Fe(III) oxide reduction kinetics. Geochimica Et Cosmochimica Acta 68: 3205-3216.

Roden EE, Sobolev D, Glazer B, Luther GW. (2004). Potential for microscale bacterial Fe redox cycling at the aerobic-anaerobic interface. Geomicrobiol $J \mathbf{2 1}$ : 379-391.

Sobolev D, Roden EE. (2002). Evidence for rapid microscale bacterial redox cycling of iron in circumneutral environments. Antonie Van Leeuwenhoek International Journal of General and Molecular Microbiology 81: $587-597$.

Sobolev D, Roden EE. (2004). Characterization of a neutrophilic, chemolithoautotrophic Fe(II)-oxidizing beta-proteobacterium from freshwater wetland sediments. Geomicrobiol J 21: 1-10.

Søgaard EG, Aruna R, Abraham-Peskir J, Koch CB. (2001). Conditions for biological precipitation of iron by Gallionella ferruginea in a slightly polluted ground water. Appl Geochem 16: 1129-1137.

Straub KL, Benz M, Schink B. (2001). Iron metabolism in anoxic environments at near neutral pH. FEMS Microbiol Ecol 34: 181-186.

Weiss JV, Rentz JA, Plaia T, Neubauer SC, Merrill-Floyd M, Lilburn $\mathrm{T}$ et al. (2007). Characterization of 
neutrophilic Fe(II)-oxidizing bacteria isolated from the rhizosphere of wetland plants and description of Ferritrophicum radicicola gen. nov sp nov., and Sideroxydans paludicola sp nov. Geomicrobiol J 24: 559-570.

Widdel F, Schnell S, Heising S, Ehrenreich A, Assmus B, Schink B. (1993). Ferrous iron oxidation by anoxygenic phototrophic bacteria. Nature 362: 834-836.
Winderl C, Anneser B, Griebler C, Meckenstock RU, Lueders T. (2008). Depth-resolved quantification of anaerobic toluene degraders and aquifer microbial community patterns in distinct redox zones of a tar oil contaminant plume. Appl Environ Microbiol 74: 792-801.

Zhou JZ, Bruns MA, Tiedje JM. (1996). DNA recovery from soils of diverse composition. Appl Environ Microbiol 62: $316-322$. 